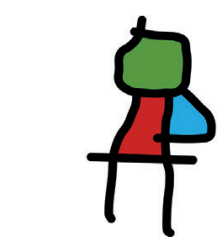

SEICAIP

\section{Allergologia et immunopathologia}

Sociedad Española de Inmunología Clínica, Alergología y Asma Pediátrica

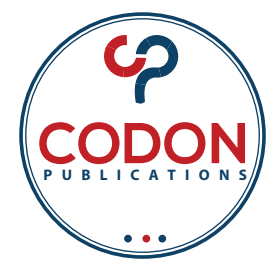

\title{
Inhaled corticosteroids may have a protective effect against coronavirus infection
}

\author{
Alicia Armentia ${ }^{a *}$, Sara Fernández Cortés ${ }^{a}$, Angela Moro Simóna , Blanca Martín-Armentia ${ }^{a * *}$, \\ Sara Martín-Armentia ${ }^{b}$, Daniel Ramos Polloc, Juan Claudio Navarro Cañadasc, \\ Javier Visa Cañoc, Álvaro Sanz Rubiales ${ }^{d}$, Joaquín Rodriguez Fra ${ }^{d}$, Sara García Vaqueroe, \\ Marta Cano Pazose ${ }^{e}$ Jesús Galán de la Calle e, Edgardo Raul Lowelle, Cristina Crespo Sotof, \\ Carmen Sánchez Toledanog ${ }^{\text {, Aurora Sacristán Salgado }}{ }^{\mathrm{h}}$
}

\author{
${ }^{a}$ Allergy Service, Hospital Universitario Río Hortega, Valladolid University, Valladolid, Spain \\ ${ }^{b}$ Pediatric Service, Delicias Center, Valladolid, Spain \\ cPalliatives and Home Care Service, Hospital Universitario Río Hortega, Valladolid, Spain \\ ${ }^{d}$ Oncology Service, Hospital Universitario Río Hortega, Valladolid, Spain \\ eMedicina de Familia y Comunitaria, ASVAO, Valladolid, Spain \\ ${ }^{f}$ Endocrinology Service, Hospital Universitario Río Hortega, Valladolid, Spain \\ ${ }^{3}$ Coordinadora de la Gerencia de Atención Primaria, Consejería de Sanidad, Madrid, Spain \\ hPreventive Medicine Service, Hospital Universitario Río Hortega, Valladolid, Spain \\ **Blanca Martín is a Pharmacy Technician.
}

Received 19 April 2020; Accepted 5 June 2020

Available online 2 January 2021

\section{KEYWORDS \\ allergy; \\ coronavirus; \\ nursing home; \\ asthma severe; \\ corticosteroids; \\ COVID; \\ telemedicine; \\ Th2 cell immunity; \\ coronavirus infection}

\begin{abstract}
Background: Spain has been severely affected by the COVID-19 epidemic, with 195,944 persons infected and 20,453 deaths at the time of writing. Older people with respiratory or cardiac conditions are most at risk.

Objective: The aim was to compare respiratory symptoms in nursing home residents and patients with uncontrolled asthma, who are considered vulnerable to COVID-19.

Methods: We studied 134 nursing home residents and 139 patients with uncontrolled asthma, groups vulnerable to COVID-19. Demographic characteristics, clinical manifestations, outcomes, key laboratory results, and radiological images were collected from medical records. COVID-19 infection was detected by polymerase chain reaction (PCR).

Results: Thirteen $(9.3 \%)$ patients with uncontrolled asthma, all receiving inhaled corticosteroids were infected by COVID-19. Eighty (60\%) nursing home residents were infected; only 28 , all of whom had received inhaled corticosteroids, had a good prognosis.
\end{abstract}

${ }^{*}$ Corresponding author: Alicia Armentia. Sección de Alergia, Hospital Universitario Rio Hortega, Dulzaina 2, 47012 Valladolid, Spain. Email address: aliciaarmentia@gmail.com 
Conclusions: Early treatment with inhaled corticosteroids may be helpful in COVID-19 infection. Persons with an allergy might have some protective mechanisms against coronavirus. (c) 2021 Codon Publications. Published by Codon Publications.

\section{Introduction}

Currently, there are 195,944 persons with COVID-19 infection and 20,453 deaths in Spain, which has followed expert contingency plans and recommendations and Chinese and Korean evidence. ${ }^{1-4}$ Most allergy/immunology care has been postponed and allergy specialists seconded to nursing homes and palliative care. The aim was to compare respiratory symptoms in nursing home residents and patients with uncontrolled asthma, persons with a high risk of COVID-19 infection due to repeated hospitalizations after infections.

\section{Methods}

We carried out a cross-sectional observational descriptive study of COVID-19 infection in uncontrolled asthmatics and nursing home residents from the Valladolid Health Area. The study period was from February 29 to April 19, 2020. All patients with uncontrolled asthma (defined as severe, life-threatening attacks or frequent hospitalization, and requiring chronic corticosteroid use, according to the Spanish Guide to Asthma Management [GEMA 4.4] criteria) were recruited from the Rio Hortega University Hospital Asthma Unit and attended using a mobile app during the pandemic. A convenience sample of residents of nursing homes with possible COVID-19 infections was recruited. All participants gave signed informed consent and the study was approved by the Rio Hortega Research Ethics Committee.

\section{Study variables}

COVID-19 was diagnosed using PCR (Seegene ${ }^{\circledR}$ ). The variables collected were sociodemographic (age, sex), epidemic (socioeconomic situation, social isolation, cohabitation with an infected patient), and clinical (asthma diagnosis, comorbidities [obesity, rhinitis, polyposis, NSAID intolerance, atopy, diabetes, hypertension, heart disease], regular medication) factors. In asthma patients, we also collected the Asthma Quality of Life Questionnaire (ACT) (in patients aged $>12$ years) or Asthma Control Questionnaire (in patients aged $<12$ years) and peak expiratory flow measured using DATOSPIR PEAK-10.

The 139 patients with uncontrolled asthma (122 adults and 17 children, 84\% sensitized to environmental allergens) had previously had prick and IgE tests for aeroallergens and foods and component resolved diagnosis in severe cases. Therapy included bronchodilators, inhaled or systemic corticosteroids, antihistamines, inhaled immunotherapy, biological immunomodulators, hydroxychloroquine, azithromycin, and antiretrovirals.

\section{Statistical analysis}

The t-test was used to compare means for continuous variables and the Chi-square test to compare proportions. The odds ratio of the treatment effects on outcomes was calculated. The odds ratio expressed in the results was calculated as the ratio between the odds of achieving FEV1/CVF $>0.90$ and AQLQ $>24$ with inhaled corticosteroids compared with usual Covid-19 care. Ratios were adjusted for imbalances between the treatment arms in certain key characteristics.

\section{Results}

One hundred and thirty-nine patients with uncontrolled asthma and 134 nursing home residents completed the protocol. The mean age was $35 \pm 16.24$ years in asthmatics and $89.4 \pm 75$ years in nursing home residents $(P<0 \cdot 001): 77$ (53\%) of asthmatics and 91 (68\%) of nursing home residents were female (Table 1).

\section{Asthmatic patients}

All asthma patients were treated with inhaled corticosteroids, 82 at low doses of $\mathrm{Cl}+\mathrm{LABA}, 37$ at medium doses, and 20 at high doses. Thirteen patients were infected by COVID-19, but none became uncontrolled. No asthmatic patients sensitized to allergens $(109,78.4 \%)$ contracted

Table 1 Summary of clinical data.

\begin{tabular}{lcccc}
\hline & \multicolumn{4}{c}{ Nursing } \\
& Asthmatic home & \\
& patients & residents & P-value \\
Number of patients & 139 & 134 & \\
Age & $35 \pm 16.24$ & $89.4 \pm 75$ & \\
Female & 77 & 91 & & \\
COVID-19 positive & 13 & 80 & 0.001 \\
Laboratory abnormalities & 17 & 18 & & \\
$\quad$ and comorbidities & & & & \\
Inhaled corticosteroids & Yes & Yes & No & \\
Number of patients & 139 & 35 & 99 & \\
Symptom-free & 139 & 28 & 7 & 0.0001 \\
Deaths & 0 & 6 & 29 & 0.004 \\
Bad prognosis & 0 & 7 & 28 & \\
COVID-19 positive & 13 & 35 & 0 & \\
Sensitization to allergens & 109 & 39 & 95 & \\
Asymptomatic or controlled & 139 & 25 & 14 & 0.005 \\
Immunomodulatory therapy & 52 & 0 & & \\
$\quad$ (12 biological, 40 IIT) & & & & \\
\hline IIT: Inhalant immunotherapy. & & & &
\end{tabular}




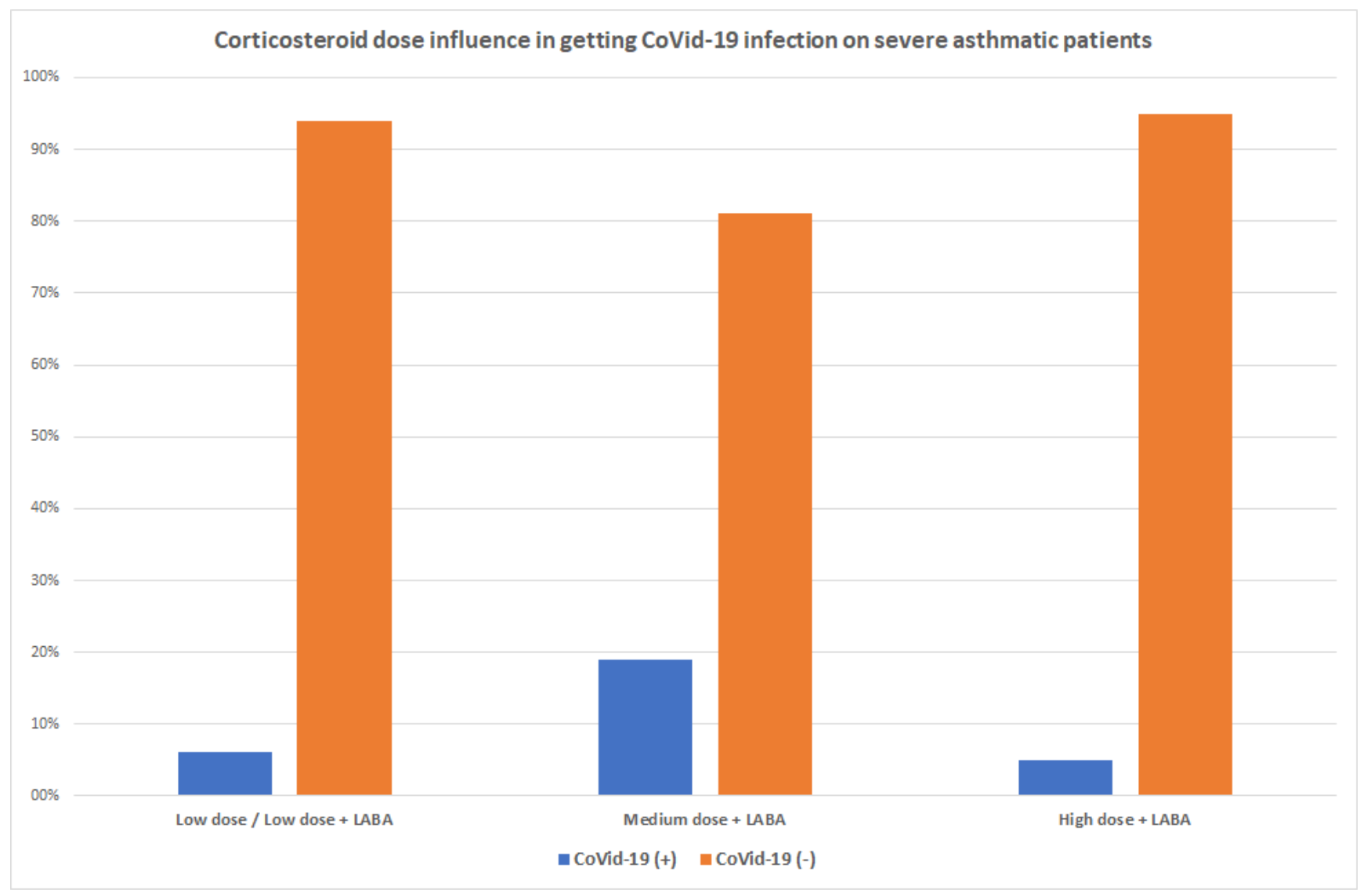

Figure 1 All asthma patients were treated with inhaled corticosteroids, 82 at low doses of $\mathrm{Cl}+\mathrm{LABA}, 37$ at medium doses, and 20 at high doses. Thirteen patients were infected with COVID-19, but none became uncontrolled.

COVID-19. Asthmatic patients treated with inhaled corticosteroids had significantly better spirometry and subjective scores $(P=0.0001)$. Eight percent of asthmatics received immunomodulatory treatment (seven mepolizumab, four omalizumab, and one benralizumab) and 40 inhalant immunotherapy with allergens. None were infected or became uncontrolled during the study period (Figure 1).

\section{Nursing home residents}

The same agreed preventive and therapeutic protocols were applied in all residences. Of the 134 nursing home residents, $80(60 \%)$ were infected by the coronavirus. Of these, $43 \%$ had previously received inhaled corticosteroids (for COPD, respiratory failure, asthma, etc.) and, of these, $80 \%$ were symptom-free, compared with $14 \%$ of deaths and $46 \%$ of severe involvement in patients not treated with inhaled corticosteroids $(\mathrm{P}<0.004)$. The two groups (35 with corticosteroids and 44 without) were comparable and homogeneous in terms of age and comorbidities (Figure 2). Thirty-nine nursing home residents had a previous allergy, of whom 25 were asymptomatic or had a good prognosis $(\mathrm{P}<0.005)$. Non-allergic patients had a worse prognosis and there were 29 deaths.

There was a significant difference in COVID-19 infections between asthma patients treated with inhaled corticosteroids and nursing home residents not treated with inhaled corticosteroids $(P=0.0001)$.

Table 1 summarizes all the results.

\section{Discussion}

Our results show asthmatic patients did not have asthma relapses, despite high pollen levels during the study period, especially the London plane $\left(400 / \mathrm{mm}^{3}\right)$ and grasses $\left(21 / \mathrm{mm}^{3}\right)$. This may be due to home confinement measures imposed after the state of alarm was declared on March 14, although some patients had allergies to indoor allergens. Patients were advised not to cease inhaled corticosteroids, which might have led to destabilization.

In the two study groups, prior allergic sensitization was associated with a favorable prognosis. A possible explanation is that COVID-19 might induce a Th2 immune response, as occurs during respiratory syncytial virus infection and coronavirus gastroenteritis, where systemic and local immune responses switched from Th1 to a Th2-based response, ${ }^{5}$ the same route used by parasites, which may generate competition for allergic patients or induce a viral defense in areas where parasitosis is endemic.

Studies show that patients with common allergic diseases do not develop severe disease.,7 Allergic disease, asthma, and COPD are not risk factors for SARS-CoV-2 


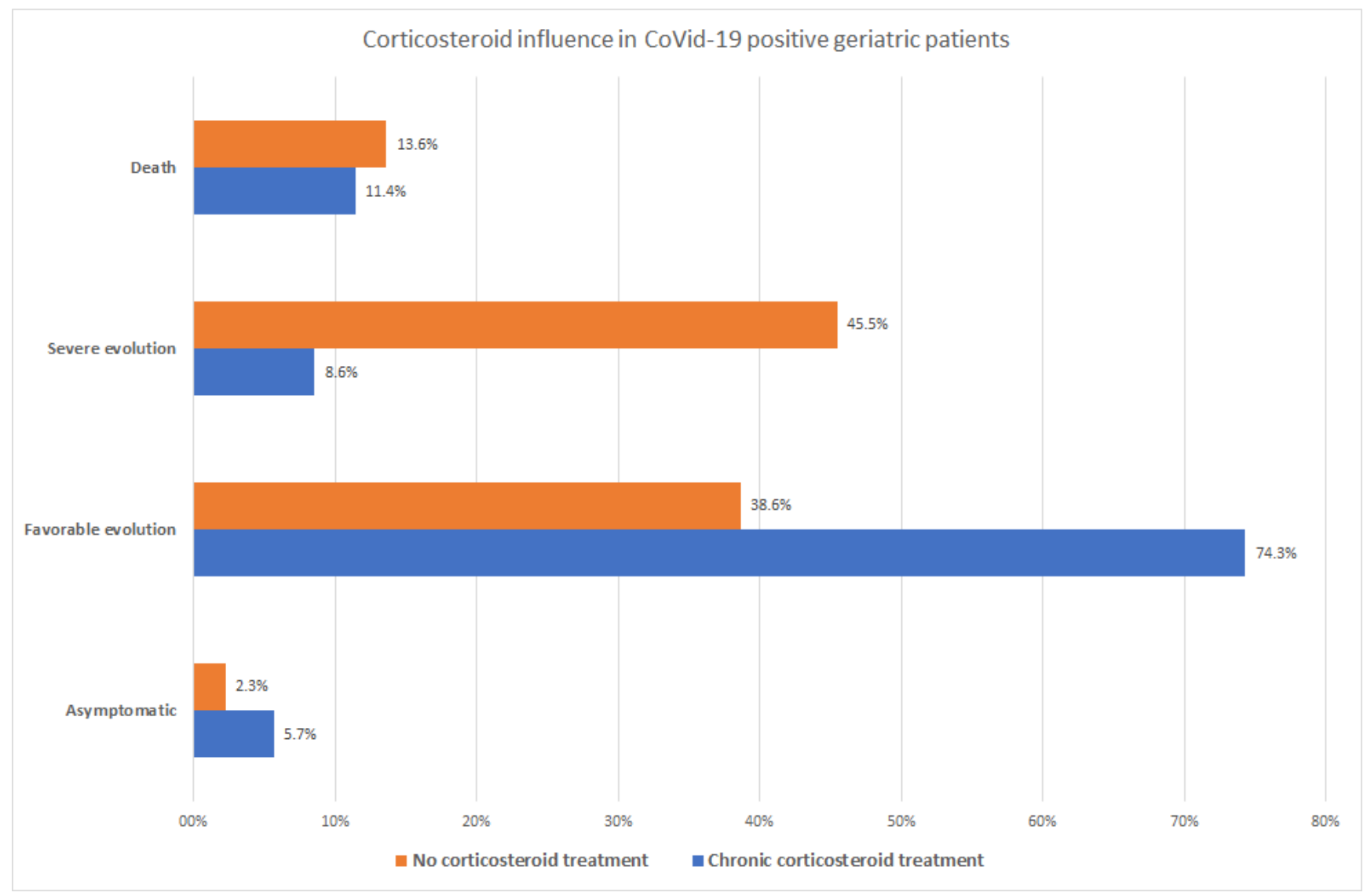

Figure 2 Of the 134 nursing home residents, 43\% received inhaled corticosteroids and, of these, 80\% evolved significantly better than patients not treated with inhaled corticosteroids.

infection but, in our patients, older age, greater comorbidity, and worse analytical results were associated with severity. COVID-19 manifests as an inflammatory syndrome due to cytokine release or hypersensitivity pneumonitis, ${ }^{8}$ and therefore, corticosteroids may be the best treatment. Inhaled corticosteroids administered at the onset of viral infection might block the inflammatory response and hypersensitivity. Like other studies, we suggest that in patients with severe COVID-19, early, short-term, lowdose methylprednisolone was beneficial $\mathbf{1 9 3 . 9 \%}$ of patients treated with low-dose inhaled corticosteroids were not infected by COVID-19) and, likewise, did not delay SARSCoV-2 RNA clearance and influence IgG antibody production. ${ }^{9}$ The WHO indicated that parenteral corticosteroid therapy (hydrocortisone $100 \mathrm{mg}$ IV) showed no benefits in the SARS and MERS epidemics, but it was applied parenterally at advanced disease stages. ${ }^{1}$ We suggest that inhaled corticosteroids might exert a targeted effect on the lungs without risk of increased viral infection. ${ }^{10}$

\section{Acknowledgments}

We thank all primary care and palliative service doctors, nurses, and technicians for their support, especially Angela Arévalo, Blanca Blanco and Natalia Gallego, and Antonio Sáez Aguado for critical review of the study.

\section{Funding}

This study was partially supported by the General Direction of Public Health, Castile and Leon (SACYL) and registered in its database as (Expt.: GRS 1952/A/19).

\section{Conflicts of interest}

The authors report no conflicts of interest.

\section{References}

1. World Health Organization Coronavirus disease 2019 (COVID-19) Situation Report. Available from: https://www.who.int/docs/ default-source/coronaviruse/20200312-sitrep-52-covid-19.pdf? sfvrsn=e2bfc9c0_2.

2. Wu Z, McGoogan JM. Characteristics of and important lessons from the coronavirus disease 2019 (COVID-19) outbreak in China: summary of a report of 72314 cases from the Chinese Center for Disease Control and Prevention. JAMA. 2020;323(13):12391242. https://doi.org/10.1001/jama.2020.2648

3. Korean Society of Infectious Diseases. Report on the epidemiological features of coronavirus disease 2019 (COVID-10) outbreak in the Republic of Korea from January 19 to March 2, 2020. J Korean Med Sci. 2020;35(10):e112. https://doi.org/10.3346/ jkms.2020.35.e112 
4. Shaker MS, Oppenheimer J, Grayson M, Stukus D, Hartog N, Hsieh E, et al. COVID-19: pandemic contingency planning for the Allergy and Immunology Clinic. J Allergy Clin Immunol Pract. 2020;8:1477. e5-1488.e5. https://doi.org/10.1016/j.jaip.2020.03.012

5. Jiang X, Hou X, Tang L, Jiang Y, Ma G, Li Y. A phase trial of the oral Lactobacillus casei vaccine polarizes Th2 cell immunity against transmissible gastroenteritis coronavirus infection. App Microbiol Biotechnol. 2016;100:7457-7469. https://doi. org/10.1007/s00253-016-7424-9

6. Dong X, Cao YY, Lu XX, Zhang JJ, Du H, Yan Yq, et al. Eleven faces of coronavirus disease 2019. Allergy. 2020;75(7):16991709. https://doi.org/10.1111/all.14289

7. Zhang JJ, Dong X, Cao YY, Yuan YD, Yang YB, Yan UQ, et al. Clinical characteristics of 140 patients infected with SARSCoV-2 in Wuhan, China, Allergy. 2020;75(7):1730-1741. https:// doi.org/10.1111/all.14238
8. Song YG, Shin HS. COVID-19. A clinical syndrome manifesting as hypersensitivity pneumonitis. Infect Chemother. 2020;52(1):110-112. https://doi.org/10.3947/ic.2020.52.1.110

9. Zheng C, Wang J, Ghuo H, Lu Z, Ma Y, Zhu X, et al. Risk adapted treatment strategy for COVID-10 patients. Int J Infect Dis. 2020;pii:S1201-9712(20)30179-X. https://doi.org/10.1016/j. ijid.2020.03.047

10. Ye Z, Wang Y, Colunga-Lozano LE, Prasad M, Tangamprnsuksan W, Rochwerg B, Yao L, Motaghi S, Couban RJ, Ghadimi M, Bala MM, Gomaa H, Fang F, Xiao Y, Guyatt GH. Efficacy and safety of corticosteroids in COVID-19 bases on evidence for COVID-19 other coronavirus infections, influenza, community-acquired pneumonia and acute respiratory distress syndrome: a systematic review and meta-analysis. CMAJ 2020;1962:756-67 https://doi.org/10.1503/cmaj. 200645 